\title{
The LEF1/CYLD axis and cIAPs regulate RIP1 deubiquitination and trigger apoptosis in selenite- treated colorectal cancer cells
}

\author{
$P \mathrm{Wu}^{1}, \mathrm{KJ} \mathrm{Shi}^{1}, \mathrm{JJ} \mathrm{An}^{1}, \mathrm{YL} \mathrm{Ci}{ }^{1}, \mathrm{~F} \mathrm{Li}{ }^{1}, \mathrm{KY} \mathrm{Hui}^{1}, \mathrm{Y} \mathrm{Yang}^{1}$ and $\mathrm{CM} \mathrm{X \textrm {u } ^ { \star , 1 }}$
}

Inhibitor-of-apoptosis protein (IAP) inhibitors have been reported to synergistically reduce cell viability in combination with a variety of chemotherapeutic drugs via targeted cellular IAP (CIAP) depletion. Here, we found that cIAP silencing sensitised colorectal cancer (CRC) cells to selenite-induced apoptosis. Upon selenite treatment, the K63-linked ubiquitin chains on receptor-interacting protein 1 (RIP1) were removed, leading to the formation of the death-inducing complex and subsequent caspase-8 activation. Although the ubiquitinases CIAP1 and cIAP2 were significantly downregulated after a 24-h selenite treatment, cylindromatosis (CYLD) deubiquitinase protein levels were marginally upregulated. Chromatin immunoprecipitation assays revealed that lymphoid enhancer factor-1 (LEF1) dissociated from the CYLD promoter upon selenite treatment, thus abolishing suppression of CYLD gene expression. We corroborated these findings in a CRC xenograft animal model using immunohistochemistry. Collectively, our findings demonstrate that selenite caused CYLD upregulation via LEF1 and cIAP downregulation, both of which contribute to the degradation of ubiquitin chains on RIP1 and subsequent caspase-8 activation and apoptosis. Importantly, our results identify a LEF1-binding site in the CYLD promoter as a potential target for combinational therapy as an alternative to clAPs.

Cell Death and Disease (2014) 5, e1085; doi:10.1038/cddis.2014.13; published online 27 February 2014

Subject Category: Cancer

Colorectal cancer $(\mathrm{CRC})$ is the third leading cause of cancerrelated death worldwide, with a 5 -year survival rate of $62 \%$ and affecting nearly a million people each year. Stage IV CRC patients have a median survival time of only 15-18 months, and more than half of all CRC patients experience local recurrence or develop distant metastases after surgery. ${ }^{1,2}$ Thus, effective drugs as well as advances in surgical management are urgently required. Selenium has been shown to possess chemopreventive and chemotherapeutic effects against multiple malignant cancers. ${ }^{3,4}$ Sodium selenite, a common form of inorganic selenium, induces typical apoptosis in cancer cells, as characterised by PARP and caspase cleavage. ${ }^{5-8}$ However, the mechanism by which these molecules are directly activated remains unknown.

Apoptosis can be initiated both by death receptors and by the mitochondria. In the death receptor-initiated extrinsic pathway, death receptor binding to their cognate ligands results in the formation of the death-inducing signalling complex (DISC) and activation of caspase-8, which induces the activation of downstream effector caspases. ${ }^{9}$ In the mitochondria-initiated intrinsic pathway, apoptosome formation by caspase-9, cytochrome $c$ and Apaf- 1 has a vital role. Extrinsic apoptosis has been reported to initiate the intrinsic mitochondrial pathway via caspase-8-mediated Bid cleavage. ${ }^{10,11}$ Whether these two pathways co-occur or whether one triggers the other in selenite-induced apoptosis remains unknown.

Receptor-interacting protein 1 (RIP1) can be modified both by lysine48-linked and lysine63-linked polyubiquitin chains. Although K48 ubiquitination promotes RIP1 degradation, K63 polyubiquitination of RIP1 on lysine 377 acts as a cell-death switch in response to TNF signalling. ${ }^{12} \mathrm{~K} 63$-polyubiquitinated RIP1, which binds to TNF and is retained at the plasma membrane, serves as a docking site to mediate NF-KB activation and thus provides survival signals. Upon TNF stimulation, RIP1 K63 ubiquitination is removed through deubiquitinase activity, resulting in the formation of a RIP1/ FADD/caspase-8 complex. The process is regulated by several ubiquitin ligases and deubiquitinating proteases. To date, only three deubiquitinating proteases that functionally impact cell death have been identified in the TNF signalling pathway, A20, cylindromatosis (CYLD) and USP2a. ${ }^{13}$ Although the mechanism by which RIP1 activates caspase-8 and triggers apoptosis after TNF stimulation has been revealed, the role of RIP1 in the induction of apoptosis by chemotherapeutic agents remains unclear.

\footnotetext{
${ }^{1}$ State Key Laboratory of Medical Molecular Biology, Department of Biochemistry and Molecular Biology, Institute of Basic Medicine Sciences and School of Basic Medicine, Peking Union Medical College and Chinese Academy of Medical Sciences, Beijing, China

${ }^{*}$ Corresponding author: CM Xu, State Key Laboratory of Medical Molecular Biology, Department of Biochemistry and Molecular Biology, Institute of Basic Medicine Sciences and School of Basic Medicine, Peking Union Medical College and Chinese Academy of Medical Sciences, Beijing 100005, China. Tel: + 86 10 69156445; Fax: + 8610 69156445; E-mail: cmxu@ibms.pumc.edu.cn Keywords: apoptosis; RIP1; clAPs; LEF1/CYLD; selenite

Abbreviations: RIP1, receptor-interacting protein 1; CYLD, cylindromatosis; K63-ub, Lys-63-linked ubiquitin; LEF1, lymphoid enhancer factor-1; cIAPs, cellular inhibitor-of-apoptosis proteins; FADD, Fas-associated protein with death domain; RIPK1, receptor-interacting protein kinase 1; FACS, fluorescence-activated cell sorting; DISC, death-inducing signalling complex; CRC, colorectal cancer; IAP, inhibitor-of-apoptosis protein; ChIP, chromatin immunoprecipitation

Received 27.11.13; revised 02.1.14; accepted 03.1.14; Edited by RA Knight
} 
Inhibitor-of-apoptosis proteins (IAPs) are a family of proteins that harbour a baculoviral IAP repeat. ${ }^{14}$ Among the IAP family members, cellular IAP (clAP)1 and clAP2 function as E3 ligases to mediate RIP1 polyubiquitination. Recently, a series of IAP inhibitors were designed and synthesised to induce apoptosis of cancer cells without affecting normal cells, ${ }^{15}$ some of which have advanced to human clinical trials. ${ }^{16}$ Because high IAP protein expression levels have been observed in CRC and correlate with a poor prognosis, targeting the IAP protein family may improve chemotherapy treatment. ${ }^{17}$

In the current study, we discovered that clAP silencing sensitised CRC cells to sodium selenite-induced apoptosis. We found that selenite triggered clAP degradation, and CYLD upregulation via the transcription factor lymphoid enhancer factor-1 (LEF1). As a result, RIP1 underwent a rapid loss of K63 ubiquitin chains and formed a complex with caspase-8 and FADD, leading to caspase-8 activation and apoptosis. Our research suggests clAPs and CYLD as targets for combinatorial therapy with selenite and indicates that this combination could potentially decrease tumour resistance as well as ameliorate the non-desirable effects of selenite alone.

\section{Results}

Caspase-8 has a vital role in selenite-induced apoptosis. Previous research from our laboratory has shown that $10 \mu \mathrm{M}$ selenite induces cell death in CRC cells, as characterised by caspase activation. To explore their response to seleniteinduced apoptosis, HCT116 and SW480 cells were treated with selenite for different durations. As shown in Figure 1a, selenite rapidly triggered caspase- 8 and caspase- 3 activation in these two cell lines, whereas caspase-7 and caspase9 were mildly activated after $24 \mathrm{~h}$ and caspase- 2 was not activated. Thus, we speculated that caspase- 8 acts as the initiator caspase in selenite-induced apoptosis. To investigate this hypothesis, CRC cells were treated with the caspase-8-specific inhibitor z-IETD-fmk before selenite treatment, and apoptosis levels were detected through Annexin V-FITC and propidium iodide double staining using fluorescence-activated cell sorting (FACS). The results showed that $20 \mu \mathrm{M}$ z-IETD-fmk inhibited selenite-induced apoptosis (Figure 1b), confirming the significance of caspase-8 in apoptotic induction following selenite treatment. In addition, silencing caspase-8 also abolished seleniteinduced apoptosis (Figure 1c). Notably, activation of downstream caspases and PARP was inhibited in cells treated with $20 \mu \mathrm{M}$ z-IETD-fmk and in cells where caspase-8 was silenced (Figures 1d and e). Moreover, cell fractionation experiments and confocal fluorescence microscopy experiments demonstrated Bid cleavage and translocation of tBid from the cytosolic fraction to the mitochondria (Figures $1 \mathrm{f}$ and $\mathrm{g}$ ), which was blocked by treatment with a caspase-8 inhibitor a

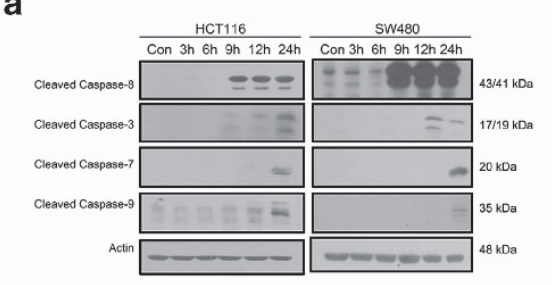

d
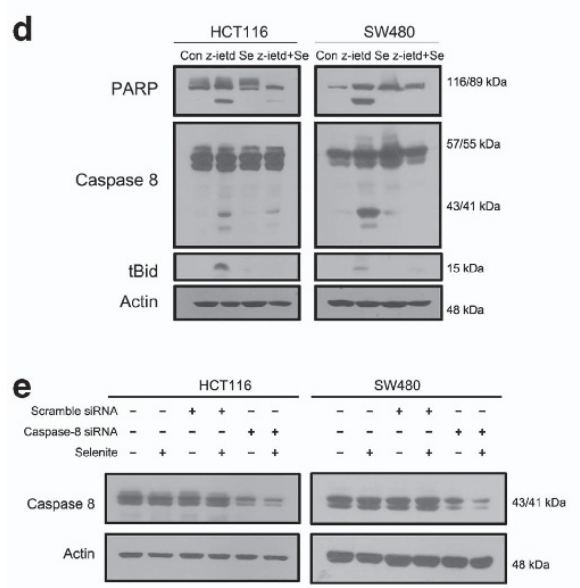

b
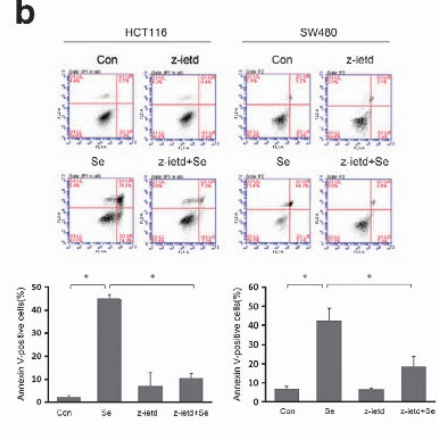
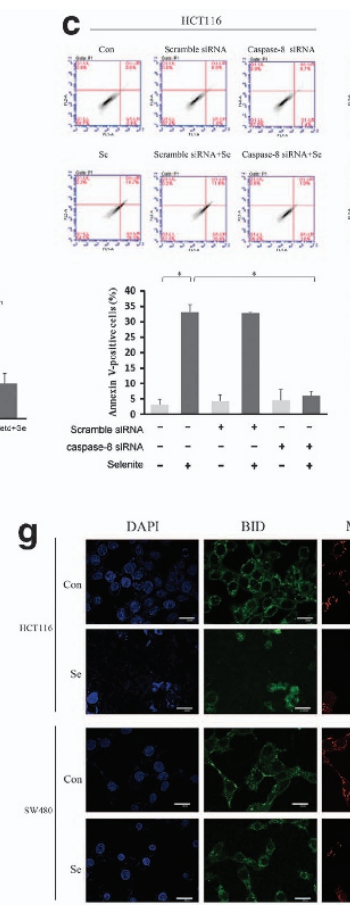
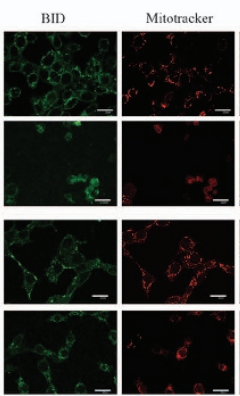
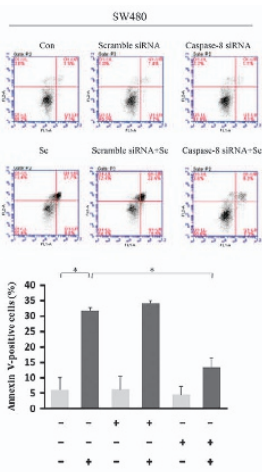

f

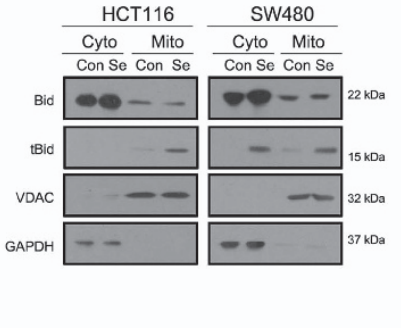

Figure 1 Selenite-induced apoptosis and activation of caspase-8. (a) Western blot analysis of activated caspases over time showing the response of HCT116 and SW480 cells to $10 \mu \mathrm{M}$ selenite from 0 to $24 \mathrm{~h}$. (b and c) HCT116 and SW480 cells were treated with the caspase-8 inhibitor Z-IETD-fmk for $1 \mathrm{~h}$ before selenite treatment or were transfected with caspase- 8 siRNA for $24 \mathrm{~h}$ before selenite treatment. The cells were then stained with Annexin V/PI to measure the ratio of apoptotic cells through FACS. (d and e) Cells were treated with z-IETD-fmk or transfected with siRNA-targeting caspase-8 and subjected to western blot analysis. (f) Selenite treatment caused the cleavage of Bid to form tBid and subsequent translocation of tBid from the cytoplasm to the mitochondria. Mitochondria were isolated after selenite treatment and immunoblotted for Bid and tBid. VDAC and GAPDH were used as mitochondrial and cytoplasmic markers, respectively. (g) Confocal analysis of Bid localization in selenite-treated HCT116 and SW480 CRC cells. Mitotracker (red) solution was added to the medium $1 \mathrm{~h}$ before harvesting cells for mitochondrial staining. The cells were collected and incubated with an antibody to detect both Bid and tBid and then stained with a FITC-conjugated secondary antibody (green). The nuclei are shown as blue signals. Scale bar, $20 \mu \mathrm{m}$. The statistical graphs are presented as the mean \pm S.D. of three independent experiments; ${ }^{*} P<0.05$ 
(Figure 1d). These findings demonstrate that caspsase- 8 is an early responder that has an important role in selenitetriggered apoptosis.

K63-specific RIP1 deubiquitination is responsible for caspase-8 activation. RIP1 has been reported to form a DISC with caspase-8 to trigger apoptosis upon TNF induction. ${ }^{18}$ To investigate the role of RIP1 in selenitetriggered caspase-8 activation, co-immunoprecipitation assays were performed using a RIP1-specific antibody. As shown in Figure 2a, caspase-8 and FADD were recruited to RIP1 following selenite stimulation. Immunofluorescence staining also confirmed the colocalisation of RIP1 and caspase- 8 in selenite-treated cells (Figure 2b). RIP1 ubiquitination involves both Lys48 and Lys63 linkages. Although Lys48 polyubiquitination of RIP1 serves as signal for protein degradation, Lys63-linked polyubiquitination is required for both NF- $\mathrm{KB}$ activation and inhibition of caspase-8-dependent apoptosis. $^{19}$ To ensure specificity, RIP1 was immunoprecipitated, and a K63-linkage-specific polyubiquitin antibody was used for detection in subsequent western blot analyses. The results revealed that endogenous RIP1 was heavily modified with K63 ubiquitin chains in colon cancer cells, whereas selenite treatment removed most of these ubiquitin chains (Figure 2c, Supplementary Figure S1a). Interestingly, in both cell lines, the length of the polyubiquitin chains on RIP1 was not equally distributed but instead accumulated at the molecular weights of 100 and $140 \mathrm{kDa}$. Taken together, these results indicated that selenite treatment induced apoptosis in colon cancer cells via removal of Lys63-linked polyubiquitin chains from RIP1and the subsequent formation of DISC by caspase-8 and FADD.

cIAPs negatively regulate selenite-induced apoptosis via RIP1. Significant clAP1 degradation upon selenite treatment has been noted in our laboratory (Figure 4a); however, the specific mechanism has remained unknown. Recently, IAP inhibitors have been reported to sensitise leukaemia cells to chemotherapy-induced apoptosis, in which clAP1 and ClAP2 act as ubiquitin ligases that specifically add K63-linked ubiquitin chains to RIP1. ${ }^{20}$ We therefore investigated the role of clAPs in selenite-induced apoptosis. FACS analysis demonstrated that the combined silencing of clAP1- and clAP2-sensitised CRC cells to selenite-induced apoptosis, suggesting a role for clAPs in inhibiting selenite-induced apoptosis via prevention of caspase-8 activation (Figure 3a). Conversely, clAP1 overexpression attenuated selenite-induced apoptosis, as supported by both FACS and western blot results (Figures $3 \mathrm{c}$ and $\mathrm{d}$ ). In addition, we corroborated the role of direct ubiquitination of RIP1 by cIAP1 in curbing seleniteinduced apoptosis (Figure 3e). Transfection of a clAP1 expression plasmid alone significantly increased RIP1 ubiquitination levels; selenite treatment removed ubiquitin proteins to levels that were still higher than in the control group, thus preventing the interaction of caspase-8 with FADD. Collectively, our study found that selenite-induced clAP1 degradation facilitated RIP1 deubiquitination and subsequent apoptosis.

RIP1 deubiquitination by CYLD promotes caspase-8 activation. Although the K63-specific deubiquitinating enzyme CYLD has previously been reported to mediate necrosis by removing K63 polyubiquitin chains from RIP1, ${ }^{21}$ its role in chemokine-induced apoptosis remains unclear. clAP levels decreased significantly upon sodium selenite treatment; however, CYLD protein levels were slightly increased (Figure 4a). Interestingly, CYLD protein levels decreased in late-phase apoptosis, despite consistently upregulated mRNA levels (Figure 4b), likely due to CYLD cleavage by caspase- 8 to prevent necrosis. ${ }^{22}$ CYLD overexpression sensitised HCT116 and SW480 cells to selenitetriggered apoptosis, as demonstrated by FACS analysis and PARP cleavage analysis. Increased caspase-8 activation was also observed in selenite-treated cells transfected with CYLD, indicating that CYLD promoted caspase- 8 activation a

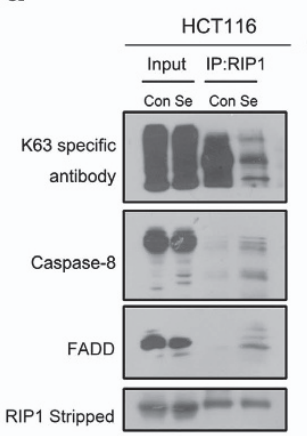

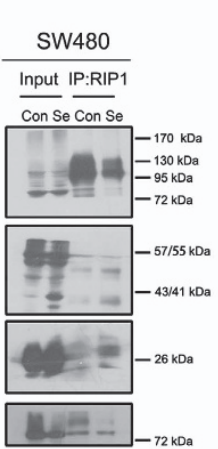

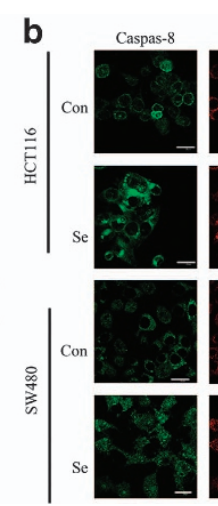

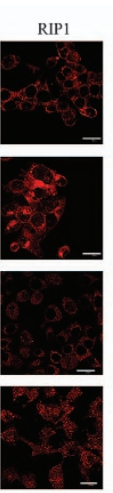
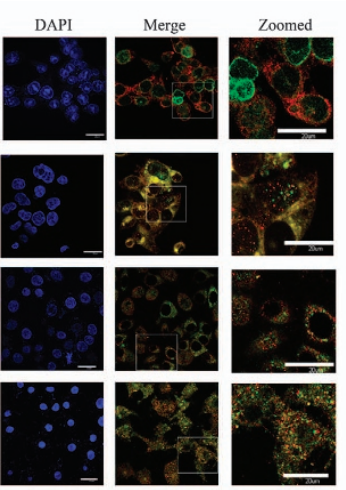

C

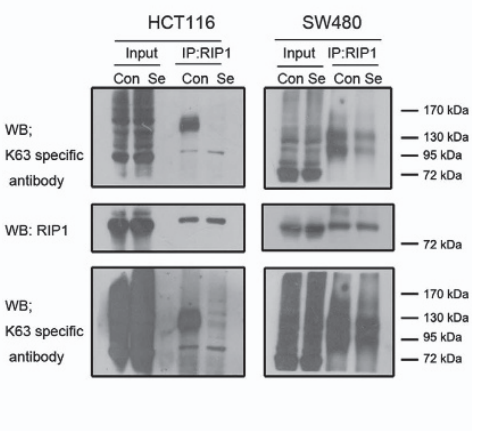

Figure 2 Selenite treatment triggers deubiquitination of K63 polyubiquitin chains on RIP1 and subsequent DISC formation. (a) RIP1 coimmunoprecipitated with caspase-8 and FADD after selenite treatment. The results from the same cell lines were acquired by incubating different antibodies to different parts of the same membrane in the same experiment. An antibody against RIP1 was applied after stripping the polyubiquitin antibody to confirm equal loading. (b) Control or selenite-treated cells were stained with antiRIP1 (CY3) and anti-caspase-8 (FITC) antibodies and subjected to confocal microscopy. The nuclei were stained with DAPI. The line furthest to the right is a magnification of the boxed area in the merged panel. Scale bar, $20 \mu \mathrm{m}$. (c) K63 ubiquitin-conjugated RIP1 was isolated by IP using an anti-RIP1 antibody followed by immunoblotting (IB) detection using anti-K63-polyubiquitin (right panel). The levels of total ubiquitinated cellular proteins were determined through direct IB in the same experiment (left panel). RIP1 was probed by IB to confirm equal loading. The results from the upper and lower panel were derived from the same experiment, with different exposure times. All experiments were performed in triplicate, and representative imagines are shown 
a
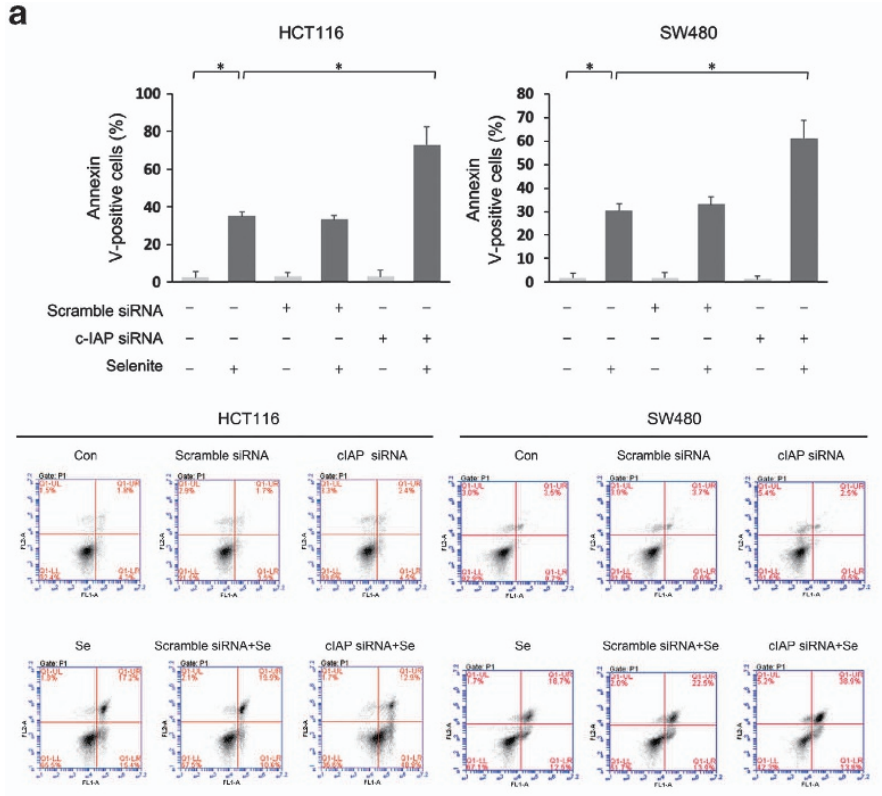

c
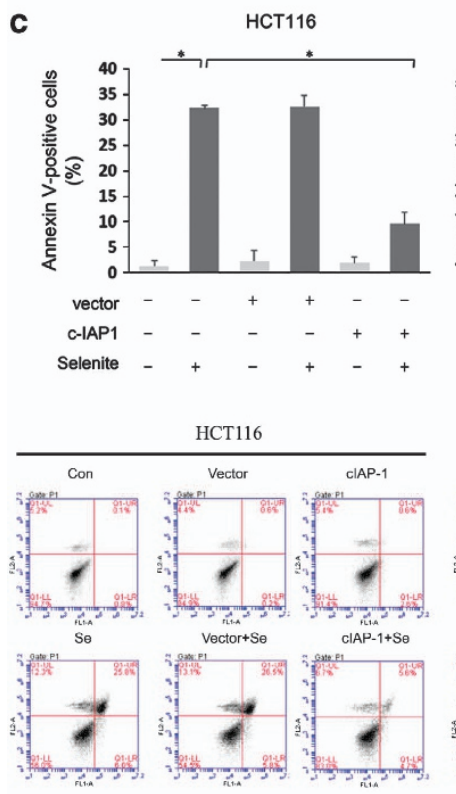
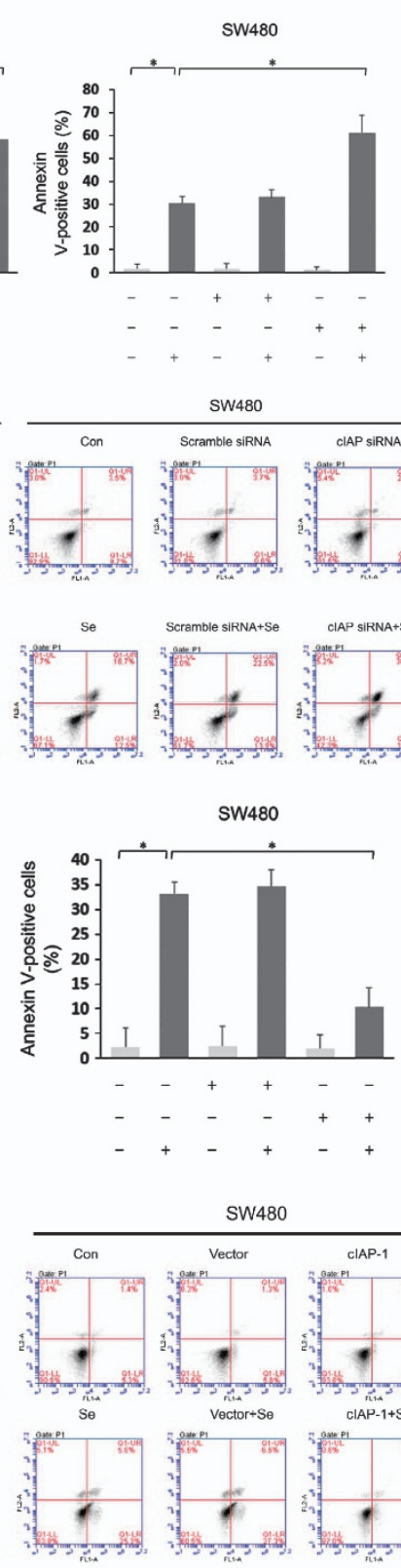

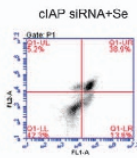

Sw480

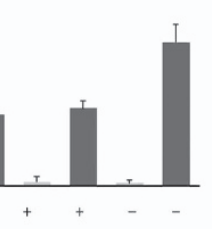

SW480
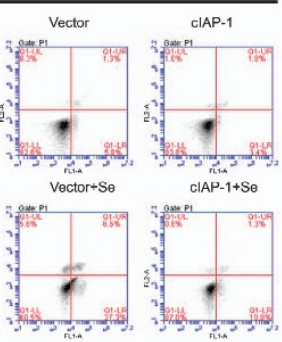

b

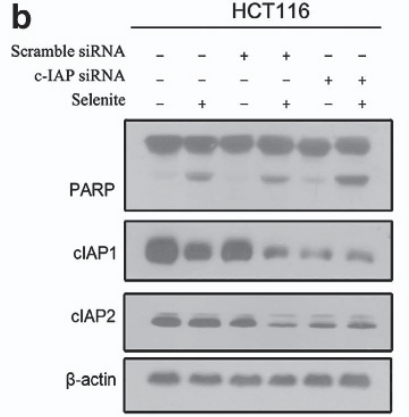

d
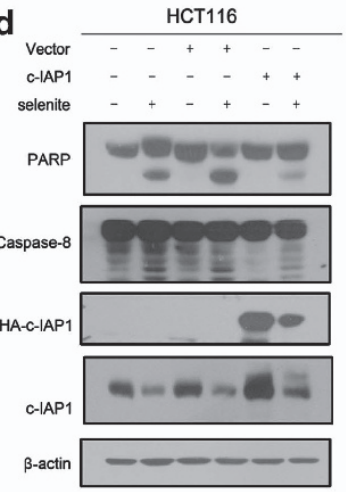

e

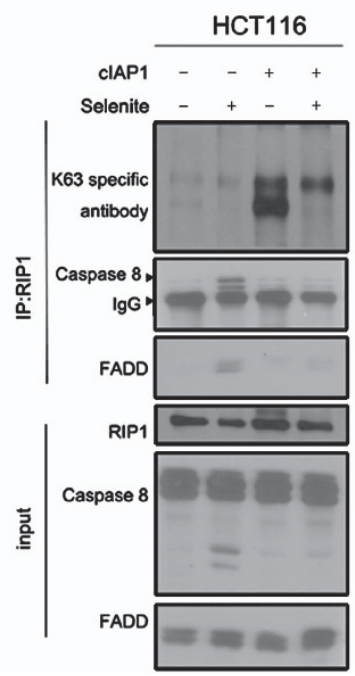

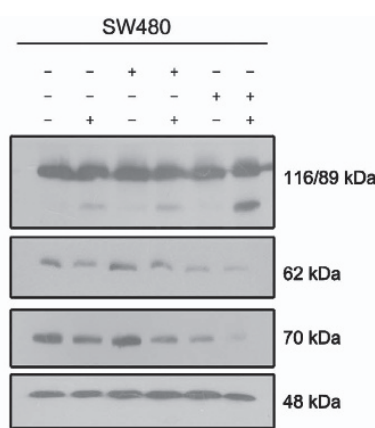
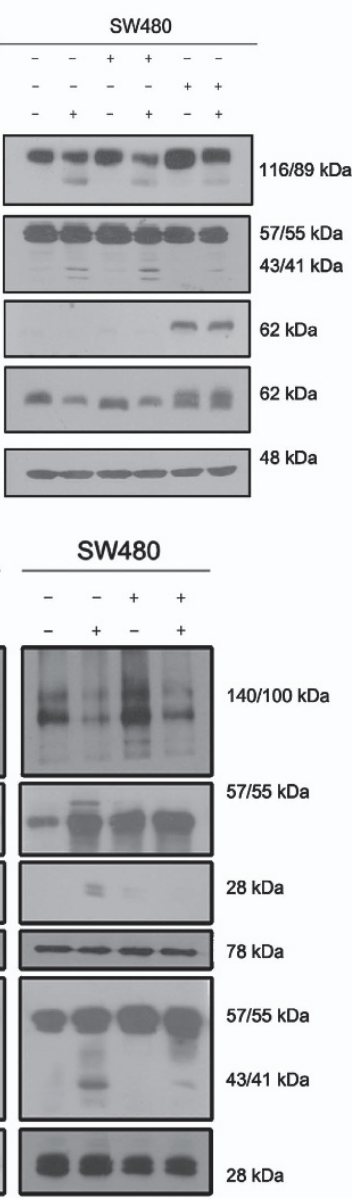

Figure 3 Selenite-induced degradation of cIAPs facilitated apoptosis by promoting DISC formation. (a and $\mathbf{b})$ After clAP silencing using an siRNA mixture containing sequences targeted to cIAP1 and cIAP2 for $24 \mathrm{~h}, \mathrm{HCT} 116$ and SW480 CRC cells were treated with $10 \mu \mathrm{M}$ selenite and subjected to FACS and western blot analysis. (c and d) HCT116 and SW480 cells were transfected with a plasmid expressing CIAP1 before selenite treatment and were then subjected to FACS and western blot analysis. (e) Coimmunoprecipitation using a RIP1 antibody was performed after transfection of CRC cells with the CIAP1 plasmid, followed by incubation with K63 polyubiquitin chain-specific antibody, caspase-8 antibody and FADD antibody

via RIP1 deubiquitination (Figures $4 c$ and d). To demonstrate that CYLD promotes selenite-induced apoptosis via RIP1 deubiquitination, HCT116 and SW480 CRC cells were transfected with a CYLD expression plasmid and used for RIP1 immunoprecipitation after selenite treatment. As shown in Figure 4e, CYLD overexpression caused RIP1 deubiquitination, which was exacerbated by selenite treatment. Taken together, these results indicate that selenite-triggered upregulation of CYLD transcription induced RIP1 deubiquitination and apoptosis.
LEF1-mediated inhibition of CYLD expression is abolished upon selenite treatment to trigger apoptosis. LEF1 has recently been reported to be a negative regulator of CYLD expression levels in leukaemia cells, and inhibitors of Wnt/beta-catenin/LEF1 signalling have been reported to induce apoptosis in leukaemia cells. ${ }^{23}$ These results inspired us to investigate the role of LEF1 in selenite-induced apoptosis. Chromatin immunoprecipitation (ChIP) assays were performed with or without selenite treatment by immunoprecipitating DNA/protein complexes using an 

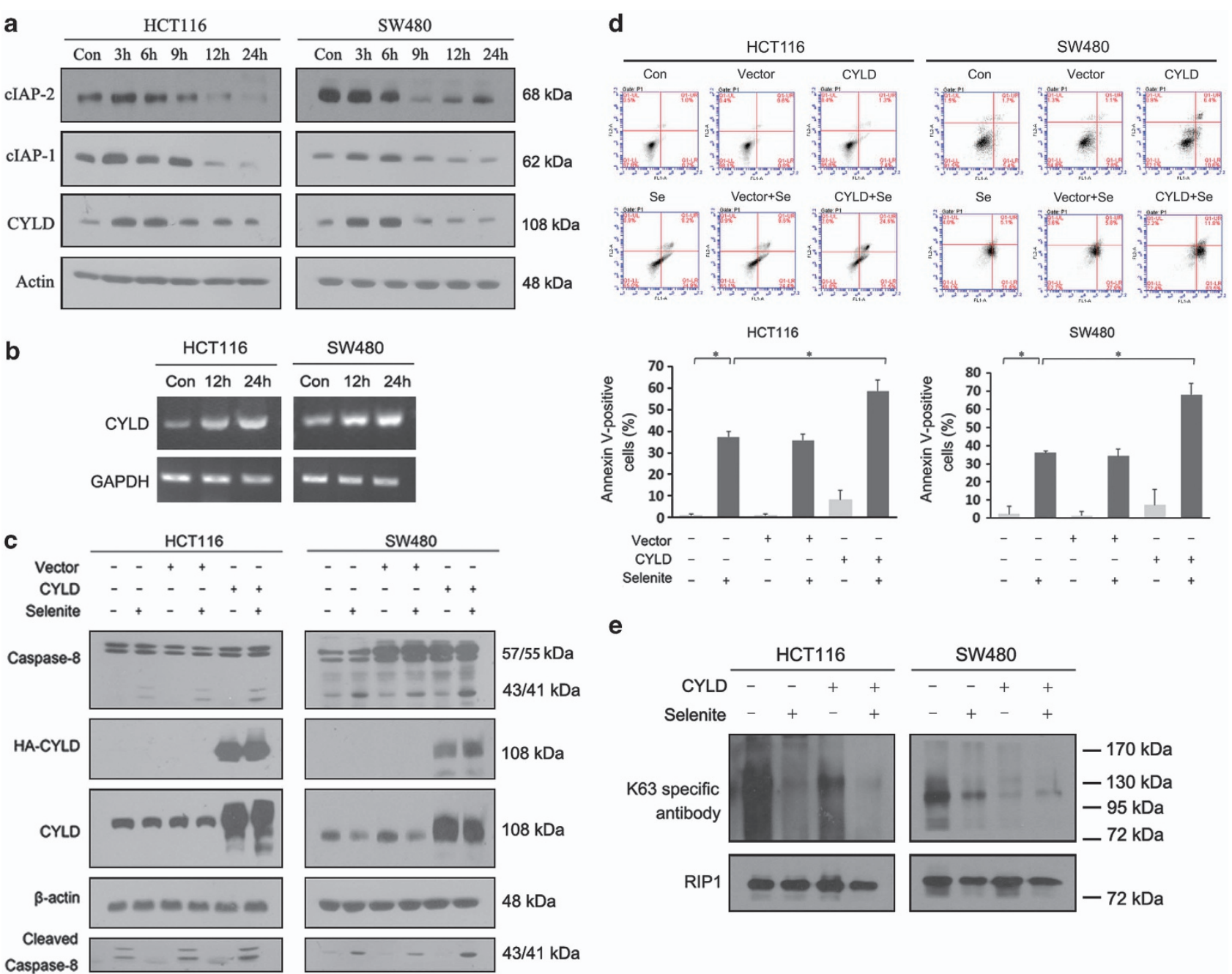

e
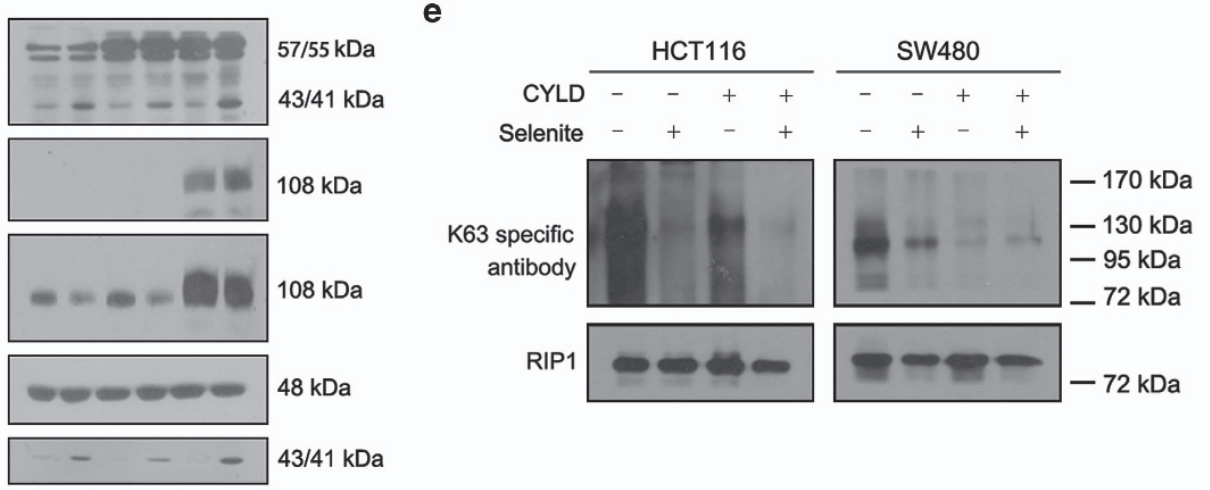

Figure 4 CYLD was responsible for apoptosis induction via RIP1. (a) Western blot analysis of clAPs and CYLD protein levels during a $0-24 \mathrm{~h}$ time course in HCT116 and SW480 cells treated with $10 \mu \mathrm{M}$ selenite. (b) Selenite increased the level of CYLD transcription in HCT116 and SW480 cells. The cells were treated with selenite for the indicated time periods followed by reverse transcription and PCR. (c and d) HCT116 and SW480 cells were transfected with the CYLD expression plasmid. Twenty-four hours post-transfection, the cells were treated with selenite $(10 \mu \mathrm{M})$ for $24 \mathrm{~h}$, and the proteins were then extracted and subjected to western blot and FACS analysis. (e) RIP1 was coimmunoprecipitated from HCT116 and SW480 cells transfected with the CYLD plasmid and treated with $10 \mu \mathrm{M}$ selenite $24 \mathrm{~h}$ after transfection. The resulting pellet was analysed by western blotting using antibodies against K63 polyubiquitin chains

anti-LEF1 antibody and then amplifying the purified DNA. The location of the LEF1-binding site within CYLD promoter and the amplified fragment are shown in Figure 5a. As shown in Figure 5b, LEF1 was recruited to the CYLD promoter in untreated CRC cells, and this interaction was abolished by selenite treatment. To confirm a negative role for LEF1 in the regulation of CYLD expression, CRC cells were transfected with siRNA-targeting LEF1 $48 \mathrm{~h}$ before selenite treatment. As shown in Figures $5 \mathrm{c}$ and d, LEF1 knockdown led to increased CYLD expression and apoptosis. Selenite-mediated disassociation of LEF1 from the CYLD promoter was not due to decreased levels of LEF1 protein or nuclear localisation (Figure 5e), implying the specific dissociation of LEF1 from the CYLD promoter upon selenite treatment.

Selenite triggers the LEF1/CYLD/cIAPs/caspase-8 signalling pathway in CRC xenograft tumours. HCT116 cells were implanted in immunodeficient nude mice to establish a CRC xenograft model over the course of 2 weeks. To treat the resulting tumours, $2 \mathrm{mg} / \mathrm{kg} /$ day selenite was administered via intraperitoneal injection. The average tumour weight in the selenite-treatment group was significantly reduced compared with the control group, which was injected with an equivalent volume of PBS (Figure 6a). Moreover, a TUNEL assay to detect apoptotic cells in situ in HCT116 xenograft tumours showed an increase in DNA fragmentation after selenite treatment (Figure $6 b$ ). Having defined the role of the LEF1/ CYLD/clAPs/caspase-8 signalling pathway in selenite-induced apoptosis in CRC cells, we evaluated the expression of these molecules after selenite treatment in vivo through western blot (Figure 6c) and immunohistochemistry (Figure 6d) assays. CIAP protein levels were downregulated, whereas CYLD was significantly upregulated in tumours from selenite-treated mice compared with PBS-treated mice. In addition, caspase-8 and PARP were cleaved and activated in tumours from selenitetreated mice. 
a

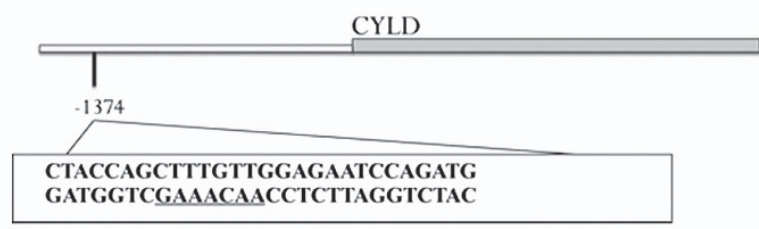

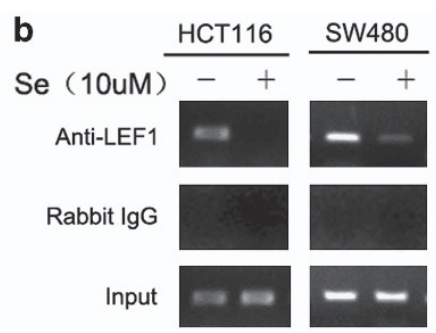

C

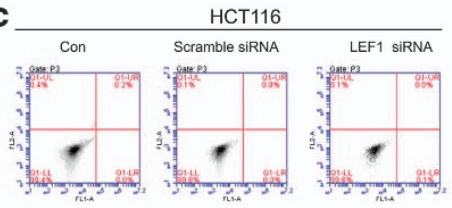

Se

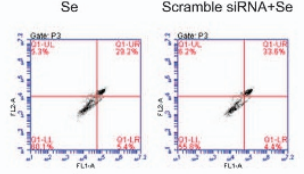

LEF1 siRNA+Se
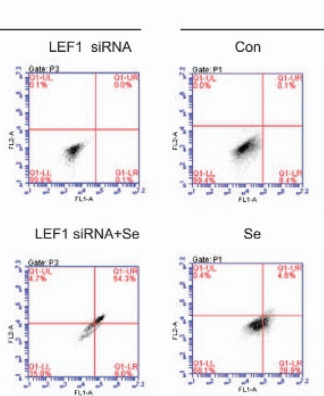

Se
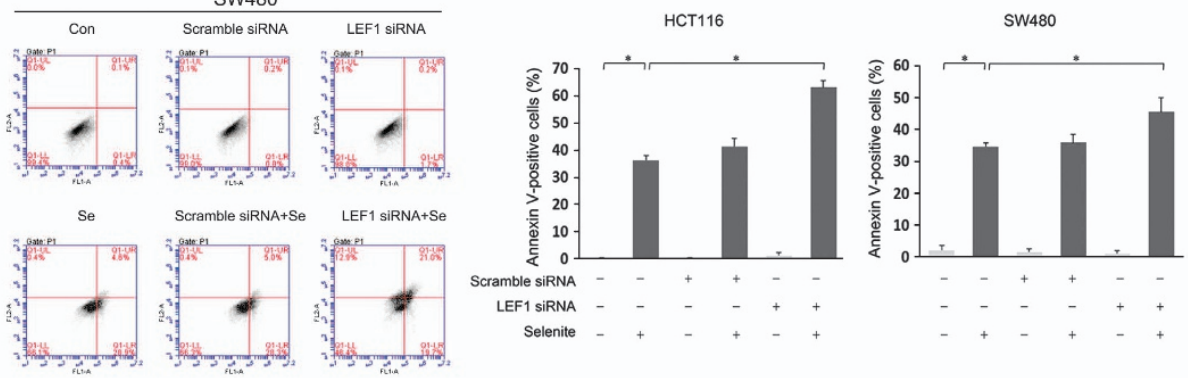

e

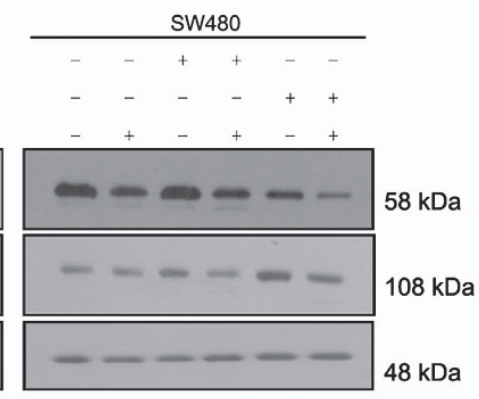

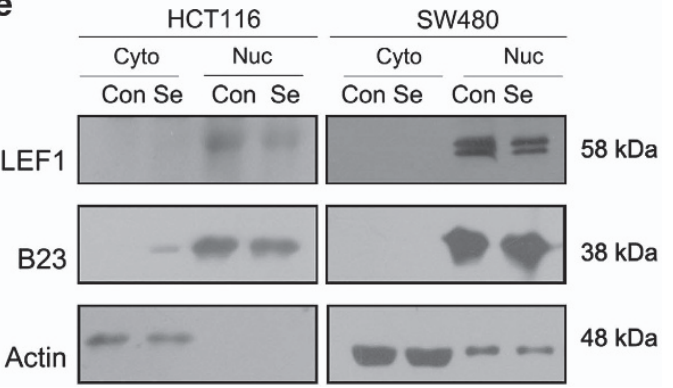

Figure 5 Suppression of CYLD expression by LEF1 is abolished upon selenite treatment. (a) A schematic representation of the LEF1-binding sites in the CYLD promoter. (b) Selenite treatment abolished LEF1 binding to the CYLD promoter. HCT116 and SW480 CRC cells were treated with selenite for $24 \mathrm{~h}$ and then subjected to ChIP analysis. (c and d) HCT116 and SW480 cells were transfected with siRNA-targeting LEF1 or scrambled control siRNA. At $48 \mathrm{~h}$ post-transfection, the cells were treated with $10 \mu \mathrm{M}$ selenite for an additional $24 \mathrm{~h}$ and then subjected to FACS and western blot analysis. (e) Overall LEF1 protein levels and distribution remained constant after selenite treatment. Nuclear and cytoplasmic proteins were fractioned from selenite-treated HCT116 and SW480 cells and subjected to western blot analysis using antibodies against LEF1. B23 and $\beta$-actin were detected to demonstrate the purity of each fraction

\section{Discussion}

Previous work has indicated that the cleavage and activation of caspases and PARP is a common feature of seleniteinduced apoptosis in a variety of cell lines. ${ }^{24,25}$ Selenite has been reported to activate multiple signalling pathways. However, it is unclear whether these pathways are initial responders, late-phase activators that enhance apoptosis or simply molecules affected by apoptosis. Because we had previously observed stable caspase activation in a variety of cell lines including CRC cells, we investigated the upstream molecules that induce apoptosis rapidly via caspase activation. CRC cells were treated with $10 \mu \mathrm{M}$ selenite for differing durations and then subjected to western blot analysis. Among the initiator caspases, caspase-8 was activated the earliest. Both caspase-8 silencing and inhibition of caspase-8 activation with the specific inhibitor z-IETD-fmk were sufficient to suppress selenite-induced apoptosis, confirming the vital role of caspase-8 in the initiation of selenite-induced apoptosis.

Selenite has also been reported to induce apoptosis via activation of the mitochondrial pathway. However, whether these two pathways co-occur or whether one triggers the other in selenite-induced apoptosis remains unknown. Here, we found that Bid is cleaved by caspase- 8 and that truncated Bid translocates to the mitochondria to launch the mitochondrial phase of apoptosis. This process has been reported to result in the release of mitochondria-derived activator of caspases (Smac) into the cytosol, which then accelerates CIAP degradation and caspase-8 activation. ${ }^{26}$ This result partially explains the sustained low cIAP levels after selenite treatment.

Antagonists of IAPs, which are in clinical trials, have been known to potentiate death receptor-induced apoptosis by promoting caspase-8 activation. Because ligation of the death receptors TNF and TRAIL by either their cognate ligands or agonistic antibodies results in caspase-8 activation at the DISC, it follows that IAP inhibitors may enhance apoptosis by enhancing DISC formation. ${ }^{27}$ In addition, it has been recently reported that targeting IAP proteins represents a suitable strategy for priming leukaemia and glioblastoma cells to undergo chemotherapy-induced apoptosis. ${ }^{20,28}$ Whether targeting IAP proteins can potentiate chemotherapy-triggered apoptosis in CRC cells is unknown. In our study, clAP-1 and 
a

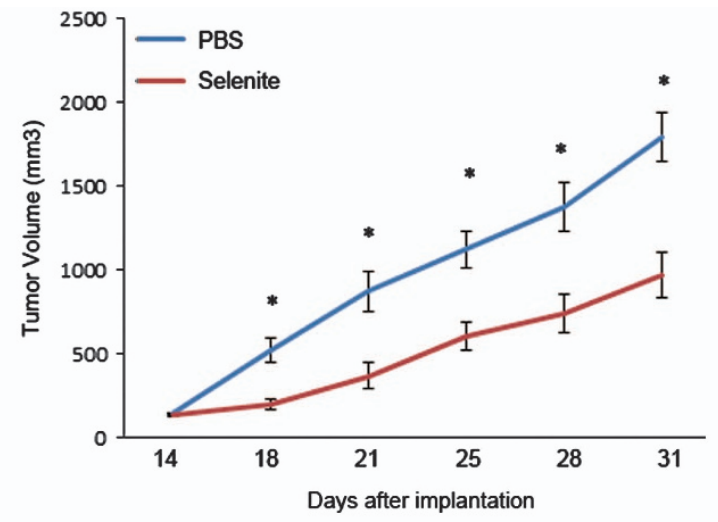

b

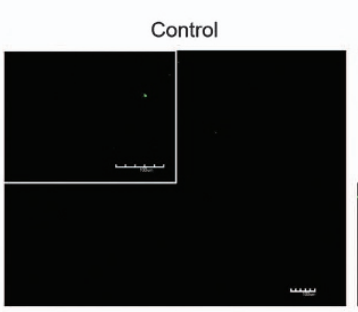

d

西

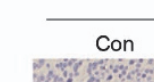

SW480
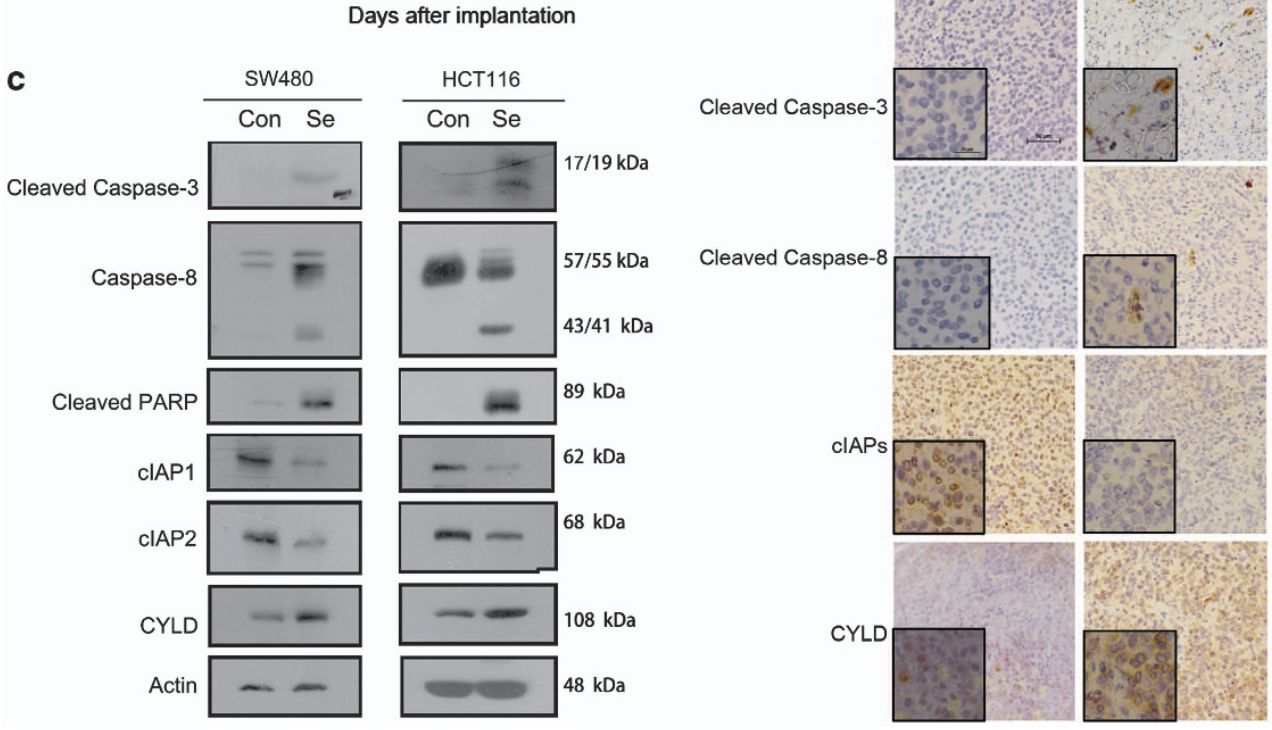
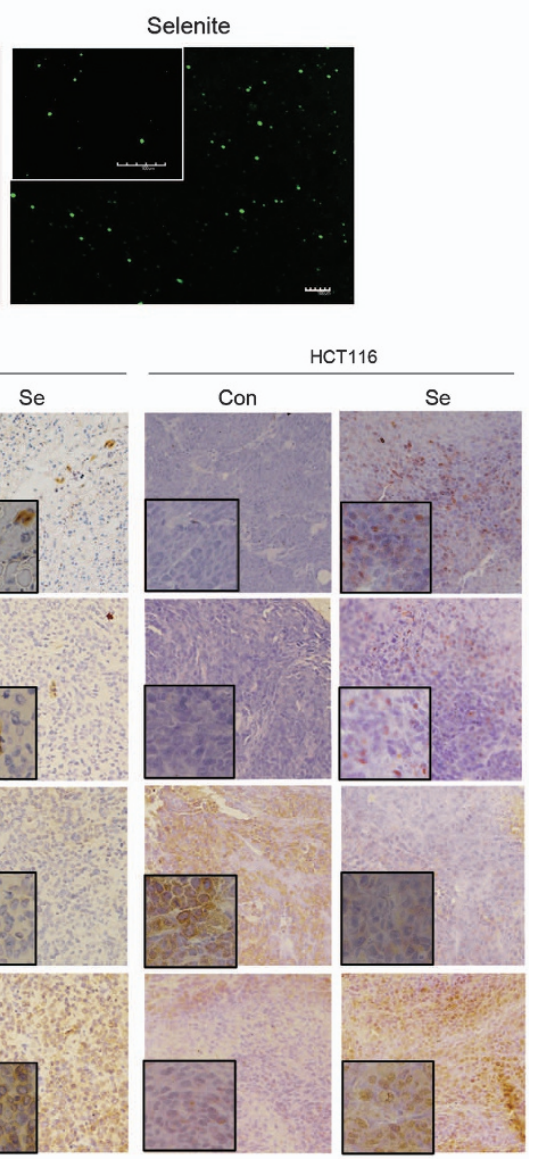

Figure 6 Selenite demonstrated antitumour activity in a colon cancer xenograft model by triggering apoptosis via the LEF1/CYLD/clAPs/caspase-8 signalling pathway. (a) Fourteen days after inoculation with HCT116 cells, nude mice (seven per group) were injected with PBS or selenite ( $2 \mathrm{mg} / \mathrm{kg} / \mathrm{d})$. Tumour volumes were calculated at the indicated intervals. The data are presented as the mean \pm S.D. (b) Representative images and quantitative analysis of in situ labelling of apoptosis cells using TUNEL assay; original magnification, $10 \times$. Scale bar, $100 \mu \mathrm{m} .{ }^{*} P<0.05$. (c) Western blot analysis of proteins extracted from tumour tissues using antibodies against related molecules as indicated. (d) Immunohistochemistry results of the colon xenograft animal model using antibodies against cleaved caspase-8, cleaved caspase-3, CYLD and clAPs (detecting both CIAP1 and clAP2). Scale bar, $50 \mu \mathrm{m}$

CIAP-2 expression levels were downregulated after selenite treatment, and clAP silencing significantly enhanced seleniteinduced apoptosis.

K48 ubiquitin chains primarily target proteins for degradation by the proteasome, whereas K63 ubiquitin chains act as signals in DNA repair, kinase activation, endocytosis and apoptosis. Immunofluorescence studies have shown that K48- and K63-linked polyubiquitin occupy distinct subcellular regions. Although K48-linked polyubiquitin coincides with proteasomes and is detected at the midbody during mitosis, K63-linked polyubiquitin speckles do not colocalise with either the Golgi complex or lysosomal markers. ${ }^{29}$ Because RIP1 can be modified by both $\mathrm{K} 48$ and $\mathrm{K} 63$ ubiquitin chains, it is vitally important to specifically detect the K63 polyubiquitin chains. Therefore, we used a K63 linkage-specific antibody after immunoprecipitation using a RIP1 antibody. Interestingly, rather than showing a distribution gradient, it appears that the number of ubiquitin proteins conjugated to RIP1 in Lys63 linkages tends to be either three or seven. This was also observed in western blot analysis data from previous experiments, where ubiquitin chains were detected after immunoprecipitation with a RIP1 antibody. ${ }^{30}$ In contrast, M1-linked polyubiquitin does not appear to have any bias in the number of ubiquitin proteins conjugated to RIP1 $1{ }^{31}$ Notably, transfection of clAP1 or CYLD expression plasmids changed the RIP1 ubiquitination levels, whereas the number of ubiquitin proteins conjugated to each RIP1 protein appeared to remain constant. The number of K63 ubiquitin proteins conjugated to RIP1 may have particular significance, and machinery that has not been previously defined may exist to regulate the precise length. The exact number of ubiquitin proteins conjugated to RIP1 in K63 linkages, and the relevant regulatory machinery require elucidation to better understand caspase-8-dependent apoptosis.

CYLD is a deubiquitinase that specifically deconjugates K63-linked polyubiquitin chains and has been reported to negatively regulate the NF- $\mathrm{KB}$ pathway as well as promote apoptosis. ${ }^{32}$ Recent studies have revealed its role in necrosis and defined a complex molecular bifurcation that controls apoptosis. ${ }^{33}$ In addition to its roles in promoting cell death and suppressing tumour formation, CYLD can also regulate diverse physiological processes including the immune 
response, inflammation, cell cycle progression, spermatogenesis and osteoclastogenesis. ${ }^{34}$ CYLD transcription is strongly reduced in $\mathrm{CRC}$ cells compared with normal tissue. ${ }^{35}$ Our research indicates that CYLD is rapidly upregulated upon selenite treatment. However, after apoptosis has been initiated, CYLD protein levels are reduced to nearly background levels, whereas CYLD mRNA levels remain upregulated. This paradoxical phenomenon can be explained by the initiation of cell apoptosis and evasion of necrosis. Upon selenite treatment, CYLD expression is increased above a certain threshold, which leads to RIP1 deubiquitination and the subsequent formation of DISC, activation of caspase-8 and apoptosis. In MEFs and Jurkat T-cells, caspase- 8 has recently been reported to process CYLD, generating either a survival signal or an apoptotic death signal. ${ }^{22}$ Therefore, we propose that CYLD protein levels decrease to evade the necrosis elicited by sustained high CYLD levels.

The TCF/LEF family, which is composed of TCF7, TCF7L1, TCF7L2 and LEF1, is a small family of DNA-binding factors. Wnt signalling triggers TCF/LEF members to switch from repression to activation by recruiting co-activator complexes. ${ }^{36}$ When interacting with $\beta$-catenin, LEF1 has been reported to behave mainly as an activator. Disruption of this interaction or inhibition of LEF1 translocation to the nucleus leads to apoptosis in leukaemia cells, implying that LEF1 may be an attractive therapeutic target for CLL therapy. ${ }^{37}$ Liu et al. found that LEF1 suppresses CYLD expression independent of $\beta$-catenin. ${ }^{23}$ In addition, LEF1 is ectopically activated in colon cancer, ${ }^{38}$ where CYLD levels are low. These data prompted us to investigate the role of LEF1 in selenite-triggered CYLD upregulation. Our study showed that LEF1 binds the CYLD promoter and suppresses endogenous CYLD levels, whereas selenite caused LEF1 to dissociate from the CYLD promoter. However, how selenite causes the dissociation of LEF1 from the CYLD promoter without affecting LEF1 nuclear localisation warrants further study. In addition, LEF1 silencing sensitised CRC cells to selenite-induced apoptosis, which provides a new avenue for enhancing chemotherapy by targeting the efficiency of LEF1 binding to the CYLD promoter.

In conclusion, our study identifies a signalling pathway that responds to selenite and rapidly triggers apoptosis. As shown in Figure 7, upon selenite treatment, clAPs were degraded, and CYLD transcription was upregulated, causing degradation of RIP1 K63 ubiquitin chains and subsequent DISC formation, caspase-8 activation and apoptosis. CYLD was negatively regulated by LEF1, and this repression was abolished upon selenite treatment. Once apoptosis was initiated, the signalling pathways that enhance clAP expression levels were inactivated, and CYLD was processed by caspase-8, all of which favoured selenite-induced apoptosis. Because clAP targeting has been successful in both cellular and clinical experiments, these findings support the further preclinical development of sodium selenite. In addition, our study implicates LEF1 as a new target for combinational treatment.

\footnotetext{
Materials and Methods

Cell lines and culture. HCT116 and SW480 CRC cells were cultured in DMEM supplemented with $10 \%$ fetal bovine serum and antibiotics $(100 \mathrm{units} / \mathrm{ml}$ penicillin and $100 \mathrm{mg} / \mathrm{ml}$ streptomycin) at $37^{\circ} \mathrm{C}$ in a $5 \% \mathrm{CO}_{2}$ humidified environment.
}

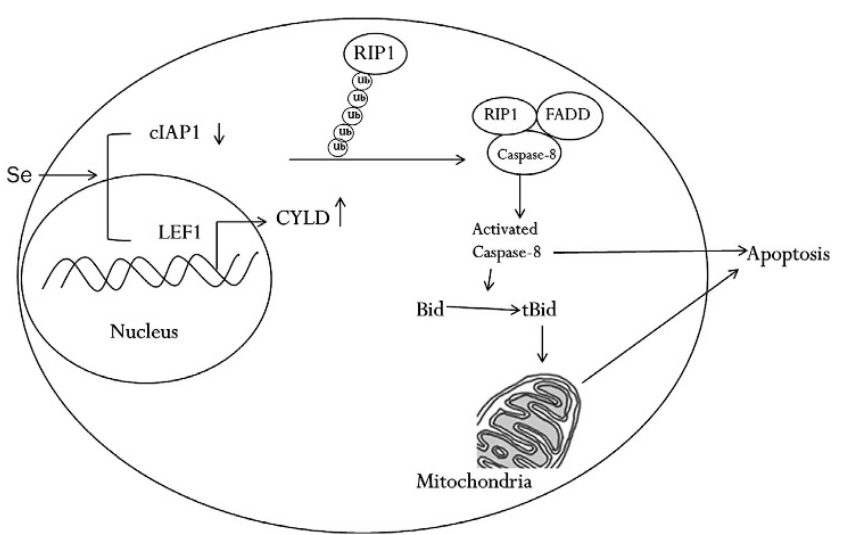

Figure 7 Schematic illustration of LEF1/CYLD and CIAPs in the modulation of RIP1 deubiquitination and triggering of apoptosis via caspase-8. Selenite treatment caused the upregulation of CYLD transcription via LEF1 and CIAP degradation, which triggered RIP deubiquitination and subsequent caspase-8 activation. Activated caspase- 8 cleaved the cytoplasmic protein Bid to generate tBid, which translocated to the mitochondria and triggered the endogenous apoptosis pathway. In addition, caspase-8 initiated the extrinsic apoptosis pathway through direct activation of executioner caspases

Reagents and antibodies. Sodium selenite, the cIAP antibody (detects both CIAP1 and CIAP2) and CYLD for immunohistochemistry analysis were purchased from Sigma-Aldrich (St. Louis, MO, USA). Antibodies for LEF1, CIAP1, CIAP2, CYLD, PARP, caspase-8, cleaved caspase-8, caspase-3, caspase-2, caspase-9, caspase-7, Bid, VDAC, HA-tag and RIP1 (for western blotting) were purchased from Cell Signaling Technology (Danvers, MA, USA). Antibodies for RIP1, caspase-8 and Bid for immunofluorescence were purchased from Santa Cruz Biotechnology, Inc. (Dallas, TX, USA). Antibodies for actin and GAPDH were purchased from Bioworld Technology (St. Louis Park, MN, USA). Caspase-8 inhibitor z-IETD-fmk was purchased from MBL (Nagoya, Japan).

Western blot analysis. Whole-cell pellets were lysed in RIPA buffer and subjected to sonication. The mitochondria were fractionated using the Mitochondria Isolation Kit for Mammalian Cells (Thermo Fisher Scientific, Rockford, IL, USA). The protein concentrations were determined using the Bradford assay. Proteins were resolved through SDS-polyacrylamide gel electrophoresis and transferred to nitrocellulose membranes, which were blocked in 5\% skim milk in Tris-buffered saline-Tween-20 and incubated overnight with antibodies at $4{ }^{\circ} \mathrm{C}$, followed by horseradish peroxidase-conjugated secondary antibodies at a 1:5000 dilution. Detection was performed using the SuperSignal West Pico Chemiluminescent Substrate (Thermo Fisher, Waltham, MA, USA).

Immunoprecipitation. The cells were harvested and washed with PBS and then lysed in RIPA buffer on ice for $1 \mathrm{~h}$. After centrifugation at $17000 \times \mathrm{g}$ for $15 \mathrm{~min}$, the supernatants were collected and adjusted to the same concentration. A $2 \%$ input sample was set aside, and either primary antibody $(2 \mu l)$ or normal immunoglobulin antibody was added to $150 \mu \mathrm{l}$ cell lysates and rotated overnight at $4^{\circ} \mathrm{C}$. Subsequently, $30 \mu$ protein $A+G$ agarose beads (Santa Cruz Biotechnology, Inc.) were added to the mixture and rotated at $4{ }^{\circ} \mathrm{C}$ for $3 \mathrm{~h}$. The target protein and its complex were collected at 4000 r.p.m. for $5 \mathrm{~min}$ at $4^{\circ} \mathrm{C}$ and washed four times with RIPA buffer.

Immunofluorescence. Cells were seeded onto coverslips and treated with selenite for $24 \mathrm{~h}$. After fixing with $5 \%$ paraformaldehyde for $10 \mathrm{~min}$, the cell membranes were permeabilised with $0.2 \%$ Triton for $30 \mathrm{~min}$. Nonspecific binding was blocked with $1 \%$ BSA for $1 \mathrm{~h}$, and the cells were stained with the indicated antibodies at $4{ }^{\circ} \mathrm{C}$ overnight and with CY3- or FITC-conjugated secondary antibodies for $1 \mathrm{~h}$ at room temperature. The slides were preserved using Mounting Medium with DAPI (Vectashield, Burlingame, CA, USA). Images were acquired using an Olympus laser scanning confocal FV1000 microscope (Olympus, Tokyo, Japan) and analysed using Olympus Fluoview software. 
ChIP assay. The target chromatin was extracted and immunoprecipitated using the SimpleChIP Enzymatic Chromatin IP Kit (Cell Signaling Technology, Catalogue no. 9002s) according to the manufacturer's instructions. Chromatin extracts were immunoprecipitated using an antibody against LEF1. Primers used to amplify the immunoprecipitated chromatin (sense $5^{\prime}$-TCCTTCCTCCTTATCG TATG-3'; antisense $5^{\prime}$-GGCAACACCGTTCTCATCTA-3') were synthesised by Sangon Biotech (Shanghai, China).

Plasmid transient transfection. The cells were plated onto six-well dishes at a density of $4 \times 10^{5}$ cells per well the day before transfection. A total of $4 \mu \mathrm{g}$ plasmid DNA was combined with $3 \mu$ l Lipofectamine 2000 (Invitrogen, Carlsbad, CA USA) in a volume of $500 \mu \mathrm{l}$ Opti-MEM Media (Gibco, Grand Island, NY, USA) and incubated for $20 \mathrm{~min}$ before being added to each well. HA-CYLD-WT was a gift from Stephen Elledge (Addgene, www.addgene.org; plasmid \#15506). The clAP1 plasmid was a gift from Colin Duckett (Addgene, plasmid \#38232).

siRNA transfection. CYLD siRNA ( $5^{\prime}$-AAGUACCGAAGGGAAGUAUAGtt-3'), CIAP1 siRNA (5'-UCCUGACAACUGGAGAGAAtt-3'), cIAP2 siRNA (5'-GAUUCGUU CAGAGUCUAAAtt-3'), LEF1 SiRNA (5'-GGAAAAGAUCUUCGCCGAG-3'), caspase-8 siRNA (5'-AACCUCGGGAUACUGUCUGA-3') and non-targeting control siRNA were acquired from GenePharma (Shanghai, China). Transfections were performed using Lipofectamine 2000 (Invitrogen) according to the recommended procedure. Briefly, $3 \mu \mathrm{l}$ Lipofectamine 2000 was combined with $4000 \mathrm{pmol}(20 \mu \mathrm{l}$ of a $20 \mu \mathrm{M}$ stock) siRNA in a volume of $500 \mu \mathrm{l}$ Opti-MEM Media (Gibco) and incubated for $20 \mathrm{~min}$ before being added directly to each well of six-well dish.

Flow cytometry analysis for apoptosis. The assay was performed using Alexa Fluor 488 Annexin V/Dead Cell Apoptosis Kit (Invitrogen) according to the manufacturer's instructions. The harvested cells were incubated in binding buffer with Annexin $\mathrm{V}$ and propidium iodide for $15 \mathrm{~min}$ and then analysed using an Accuri C6 flow cytometer (Accuri Cytometers Inc., Ann Arbor, MI, USA).

RT-PCR. Total RNA was extracted using TRIzol reagent (Invitrogen) according to the manufacturer's instructions. RNA was reverse transcribed using SuperRT cDNA kit (CW Biotech, Beijing, China). Primers for CYLD (forward: 5'-GAAGGTC GTGGTCAAGGT-3'; reverse: $5^{\prime}$-GAATCTGTTCTCGGTGGT-3') were synthesised by Sangon Biotech.

In vivo tumour model. HCT116 CRC cells $\left(1 \times 10^{7}\right)$ were inoculated subcutaneously into 6- to 8-week-old nude mice. Fourteen mice were used in each group. Selenite dissolved in PBS ( $2 \mathrm{mg} / \mathrm{kg} /$ day) was injected intraperitoneally into mice after 2 weeks, at which point, the tumours were palpable. The control group was injected with an equivalent volume of PBS. Tumour dimensions were measured using callipers, and the volume was calculated using the following formula: volume $=0.5 \times I \times w^{2}$, with ' ' being the maximal length and ' $w$ ' being the width. The mice were maintained and tested according to the UKCCCR Guidelines for the Welfare of Animals in Experimental Neoplasia.

Immunohistochemistry. Tissues from the HCT116 xenograft model were established as described above. An animal model for SW480 cells was established previously. ${ }^{39}$ Tissues were embedded in paraffin for immunohistochemical analysis. Tissue sections were prepared on slides, dewaxed and rehydrated in xylene and graded alcohols. Antigen retrieval was achieved by heating the slides in a $95^{\circ} \mathrm{C}$ water bath with $0.01 \mathrm{~mol} / \mathrm{l}$ citrate buffer at $\mathrm{pH} 6.0$ for $20 \mathrm{~min}$. Endogenous peroxidase activity was quenched by incubation in $3 \%$ hydrogen peroxide solution (Zhongshan Gold Bridge, Beijing, China). The slides were incubated with primary antibodies overnight at $4{ }^{\circ} \mathrm{C}$. The samples were incubated with a streptavidinperoxidase complex for $1 \mathrm{~h}$ at room temperature. Diaminobenzidine working solution was applied, and the slides were counterstained with haematoxylin.

Statistical analyses. Each experiment was repeated at least three times. For the quantitative analyses represented in the histograms, the values are expressed as the mean \pm S.D. The significance of differences between mean values was assessed using Student's t-test. All computations were calculated using Microsoft Excel.

\section{Conflict of Interest}

The authors declare no conflict of interest.
Acknowledgements. We thank Pan Lin and Gao Yunzou for sharing their expertise in IHC. We thank Chen Kangmei, Wu Jinru and Jiang Qian for their assistance. This work was supported by the National Natural Science Foundation of China (Grant No: 31170788, 31340037), the National Natural Science Foundation for Young Scholars of China (Grant No: 31101018), the Research Fund for the Doctoral Program of Higher Education of China (Grant No: 20091106110025) and the National Laboratory Special Fund (Grant No: 2060204).

1. Wang $Q$, Wang $X$, Evers BM. Induction of clAP-2 in human colon cancer cells through $P K C$ delta/NF-kappa B. J Biol Chem 2003; 278: 51091-51099.

2. Yao Y, Li L, Huang X, Gu X, Xu Z, Zhang Y et al. SERPINA3K induces apoptosis in human colorectal cancer cells via activating the Fas/FasL/caspase-8 signaling pathway. FEBS J 2013; 280: 3244-3255.

3. Brozmanova J. [Selenium and cancer: from prevention to treatment]. Klin Onkol 2011; 24: $171-179$

4. Rayman MP. Selenium and human health. Lancet 2012; 379: 1256-1268.

5. Luo H, Yang Y, Huang F, Li F, Jiang Q, Shi K et al. Selenite induces apoptosis in colorectal cancer cells via AKT-mediated inhibition of beta-catenin survival axis. Cancer Lett 2012; 315: $78-85$

6. Chen P, Wang L, Li N, Liu Q, Ni J. Comparative proteomics analysis of sodium seleniteinduced apoptosis in human prostate cancer cells. Metallomics 2013; 5: 541-550.

7. Kralova V, Brigulova K, Cervinka M, Rudolf E. Antiproliferative and cytotoxic effects of sodium selenite in human colon cancer cells. Toxicol In Vitro 2009; 23: 1497-1503.

8. Shi K, Jiang Q, Li Z, Shan L, Li F, An J et al. Sodium selenite alters microtubule assembly and induces apoptosis in vitro and in vivo. J Hematol Oncol 2013; 6 : 7.

9. Fulda S, Debatin KM. Extrinsic versus intrinsic apoptosis pathways in anticancer chemotherapy. Oncogene 2006; 25: 4798-4811.

10. Beaudouin J, Liesche C, Aschenbrenner S, Horner M, Eils R. Caspase-8 cleaves its substrates from the plasma membrane upon CD95-induced apoptosis. Cell Death Differ 2013; 20: 599-610.

11. Schug ZT, Gonzalvez F, Houtkooper RH, Vaz FM, Gottlieb E. BID is cleaved by caspase-8 within a native complex on the mitochondrial membrane. Cell Death Differ 2011; 18: 538-548.

12. O'Donnell MA, Legarda-Addison D, Skountzos P, Yeh WC, Ting AT. Ubiquitination of RIP1 regulates an NF-kappaB-independent cell-death switch in TNF signaling. Curr Biol 2007; 17: 418-424.

13. Mahul-Mellier AL, Pazarentzos E, Datler C, Iwasawa R, AbuAli G, Lin B et al. De-ubiquitinating protease USP2a targets RIP1 and TRAF2 to mediate cell death by TNF. Cell Death Differ 2012; 19: 891-899.

14. Wei Y, Fan T, Yu M. Inhibitor of apoptosis proteins and apoptosis. Acta Biochim Biophys Sin (Shanghai) 2008; 40: 278-288.

15. Varfolomeev E, Blankenship JW, Wayson SM, Fedorova AV, Kayagaki N, Garg P et al. IAP antagonists induce autoubiquitination of c-IAPs, NF-kappaB activation, and TNFalphadependent apoptosis. Cell 2007; 131: 669-681.

16. Flygare JA, Beresini M, Budha N, Chan H, Chan IT, Cheeti S et al. Discovery of a potent small-molecule antagonist of inhibitor of apoptosis (IAP) proteins and clinical candidate for the treatment of cancer (GDC-0152). J Med Chem 2012; 55: 4101-4113.

17. Miura K, Fujibuchi W, Ishida K, Naitoh T, Ogawa H, Ando T et al. Inhibitor of apoptosis protein family as diagnostic markers and therapeutic targets of colorectal cancer. Surg Today 2011; 41: 175-182.

18. Micheau O, Tschopp J. Induction of TNF receptor I-mediated apoptosis via two sequential signaling complexes. Cell 2003; 114: 181-190.

19. Festjens N, Vanden Berghe T, Cornelis S, Vandenabeele P. RIP1, a kinase on the crossroads of a cell's decision to live or die. Cell Death Differ 2007; 14: 400-410.

20. Loder S, Fakler M, Schoeneberger H, Cristofanon S, Leibacher J, Vanlangenakker $\mathrm{N}$ et al. RIP1 is required for IAP inhibitor-mediated sensitization of childhood acute leukemia cells to chemotherapy-induced apoptosis. Leukemia 2012; 26: 1020-1029.

21. Massoumi R. Ubiquitin chain cleavage: CYLD at work. Trends Biochem Sci 2010; 35 392-399.

22. O'Donnell MA, Perez-Jimenez E, Oberst A, Ng A, Massoumi R, Xavier R et al. Caspase 8 inhibits programmed necrosis by processing CYLD. Nat Cell Biol 2011; 13: 1437-1442.

23. Liu P, Xu B, Shen W, Zhu H, Wu W, Fu Y et al. Dysregulation of TNFalpha-induced necroptotic signaling in chronic lymphocytic leukemia: suppression of CYLD gene by LEF1. Leukemia 2012; 26: 1293-1300.

24. Luo H, Yang Y, Duan J, Wu P, Jiang Q, Xu C. PTEN-regulated AKT/FoxO3a/Bim signaling contributes to reactive oxygen species-mediated apoptosis in selenite-treated colorectal cancer cells. Cell Death Dis 2013; 4: e481.

25. Park SH, Kim JH, Chi GY, Kim GY, Chang YC, Moon SK et al. Induction of apoptosis and autophagy by sodium selenite in A549 human lung carcinoma cells through generation of reactive oxygen species. Toxicol Lett 2012; 212: 252-261.

26. Cristofanon S, Fulda S. ABT-737 promotes tBid mitochondrial accumulation to enhance TRAIL-induced apoptosis in glioblastoma cells. Cell Death Dis 2012; 3: e432.

27. Fakler M, Loeder S, Vogler M, Schneider K, Jeremias I, Debatin KM et al. Small molecule XIAP inhibitors cooperate with TRAIL to induce apoptosis in childhood acute leukemia cells and overcome Bcl-2-mediated resistance. Blood 2009; 113: 1710-1722. 
28. Wagner L, Marschall V, Karl S, Cristofanon S, Zobel K, Deshayes K et al. Smac mimetic sensitizes glioblastoma cells to Temozolomide-induced apoptosis in a RIP1- and NF-kappaB-dependent manner. Oncogene 2013; 32: 988-997.

29. Newton K, Matsumoto ML, Wertz IE, Kirkpatrick DS, Lill JR, Tan J et al. Ubiquitin chain editing revealed by polyubiquitin linkage-specific antibodies. Cell 2008; 134: 668-678.

30. Xu G, Tan X, Wang H, Sun W, Shi Y, Burlingame S et al. Ubiquitin-specific peptidase 21 inhibits tumor necrosis factor alpha-induced nuclear factor kappaB activation via binding to and deubiquitinating receptor-interacting protein 1. J Biol Chem 2010; 285: 969-978.

31. Gerlach B, Cordier SM, Schmukle AC, Emmerich CH, Rieser E, Haas TL et al. Linear ubiquitination prevents inflammation and regulates immune signalling. Nature 2011; 471: 591-596.

32. Brummelkamp TR, Nijman SM, Dirac AM, Bernards R. Loss of the cylindromatosis tumour suppressor inhibits apoptosis by activating NF-kappaB. Nature 2003; 424: 797-801.

33. Hitomi J, Christofferson DE, Ng A, Yao J, Degterev A, Xavier RJ et al. Identification of a molecular signaling network that regulates a cellular necrotic cell death pathway. Cell 2008; 135: $1311-1323$

34. Sun SC. CYLD: a tumor suppressor deubiquitinase regulating NF-kappaB activation and diverse biological processes. Cell Death Differ 2010; 17: 25-34.

35. Hellerbrand C, Bumes E, Bataille F, Dietmaier W, Massoumi R, Bosserhoff AK. Reduced expression of CYLD in human colon and hepatocellular carcinomas. Carcinogenesis 2007; 28: $21-27$.
36. Mao CD, Byers SW. Cell-context dependent TCF/LEF expression and function: alternative tales of repression, de-repression and activation potentials. Crit Rev Eukaryot Gene Expr 2011; 21: 207-236.

37. Gandhirajan RK, Staib PA, Minke K, Gehrke I, Plickert G, Schlosser A et al. Small molecule inhibitors of Wnt/beta-catenin/lef-1 signaling induces apoptosis in chronic lymphocytic leukemia cells in vitro and in vivo. Neoplasia 2010; 12: 326-335.

38. Hovanes K, Li TW, Munguia JE, Truong T, Milovanovic T, Lawrence Marsh J et al. Beta-catenin-sensitive isoforms of lymphoid enhancer factor-1 are selectively expressed in colon cancer. Nat Genet 2001; 28: 53-57.

39. Huang F, Nie C, Yang Y, Yue W, Ren Y, Shang Y et al. Selenite induces redox-dependent Bax activation and apoptosis in colorectal cancer cells. Free Radic Biol Med 2009; 46 1186-1196.

(c) (1) (2) Cell Death and Disease is an open-access journal published by Nature Publishing Group. This work is

licensed under a Creative Commons Attribution-NonCommercialShareAlike 3.0 Unported License. To view a copy of this license, visit http://creativecommons.org/licenses/by-nc-sa/3.0/

Supplementary Information accompanies this paper on Cell Death and Disease website (http://www.nature.com/cddis) 